\title{
Electronically Switchable Bandpass Filters Using Loaded Stepped-Impedance Resonators
}

\author{
Shih-Fong Chao, Chao-Huang Wu, Zou-Ming Tsai, Student Member, IEEE, Huei Wang, Fellow, IEEE, and
} Chun Hsiung Chen, Fellow, IEEE

\begin{abstract}
Stepped-impedance resonators with diodes loaded at one end are used to develop switchable bandpass filters in this paper. The loaded diodes are used to switch the resonance conditions of the stepped-impedance resonators. The equations for resonance conditions of the stepped-impedance resonators with different loads at one end are derived and discussed. With these derived equations, the switchable filters can be easily designed and synthesized using the coupled-resonator filter theory. When the switchable filter is switched on, a bandpass filter response with a wide stopband rejection is achieved by making the on-state coupled resonators have the same fundamental resonant frequency, but different higher order resonant frequencies. When switched off, a high and wideband isolation is obtained by properly misaligning the resonant modes of the off-state resonators. The design concept is demonstrated by two single-pole-single-throw fourth-order Butterworth-type switchable microstrip bandpass filters, which utilize two and three switched stepped-impedance resonators, respectively. Finally, a compact single-pole-double-throw switchable microstrip bandpass filter using common resonators is demonstrated for wireless communication applications.
\end{abstract}

Index Terms-Coupled-resonator bandpass filter, steppedimpedance resonator, switch, switchable filter.

\section{INTRODUCTION}

A MICROWAVE switch is a key building block at the RF front-end for time-division duplexing (TDD) communication systems. Recently, several works using passive field-effect transistors (FETs) or p-i-n diodes have been reported for microwave and millimeter-wave transceiver applications [1]-[5]. Most of these switches are based on wideband design, implying that their operating bandwidths are usually over $50 \%$ and cannot provide sharp cutoff outside the operating band. Such a wideband switch shows a poor band selectivity for a system application. Therefore, a bandpass filter will be needed to cascade with a switch to reject out-of-band signals. Planar filters are popular in millimeter-wave filter designs because they can be easily fabricated using printed circuit technology and integrated with other circuit components. However, conventional design of planar filters suffers from spurious responses in the upper stopband due to the nature of distributed elements [6], [7]. Therefore, several techniques have been proposed to resolve this problem [8]-[13].

Manuscript received July 29, 2006; revised September 8, 2006. This work was supported in part by the National Science Council under Grant NSC 94-2752-E-002-003-PAE, Grant NSC 94-2219-E-002-006, Grant NSC 94-2219-E-002-009, and Grant NSC 94-2752-E-002-001-PAE.

The authors are with the Graduate Institute of Communication Engineering and the Department of Electrical Engineering, National Taiwan University, Taipei 106, Taiwan, R.O.C. (e-mail: hueiwang@ew.ee.ntu.edu.tw).

Digital Object Identifier 10.1109/TMTT.2006.885898
From the above discussions, a switchable bandpass filter that integrates a bandpass filter function and a switch is desired to perform a bandpass filter function with wide stopband extension in the on state and provide a good isolation while in the off state. In [4], a ring resonator loaded with a p-i-n diode had been developed as a switchable filter. By mounting the p-i-n diodes across the gap at $90^{\circ}$ from the feed point, the odd modes can be switched according to different bias conditions to control the on and off states. However, it occupied a large layout size, and a high-order implementation is difficult. A coplanar waveguide-slotline switchable filter was given in [15], in which p-i-n diodes are mounted over the end of the open stubs to make the circuit switchable. In [16], a switchable microstrip bandpass filter based on quarter-wavelength short-stub structures was proposed. The quarter-wavelength resonators were replaced by inductive short-stubs shunt with passive FETs to make it switchable. However, these previously mentioned designs mainly focus on designing the performance around the passbands, which means that only the on-state filter response and off-state isolation in the vicinity of the center frequency were considered. Consequently, those designs would suffer from unwanted spurious responses and narrowband isolation in the on and off states, respectively.

In this paper, stepped-impedance resonators with diodes loaded at one end are developed as switchable resonators. The diode-loaded stepped-impedance resonators and unloaded stepped-impedance resonators are used to construct a switchable microstrip coupled-resonator bandpass filter. In the on state, the diodes are reversed-biased, and the diode-loaded stepped-impedance resonators behave like half-wavelength resonators. The loaded resonators together with unloaded ones form a half-wavelength coupled-resonator bandpass filter in the on state. Also, all resonators are designed to have staggered higher order resonant frequencies; therefore, a bandpass filter with wide stopband rejection can then be achieved. In the off state, the diodes are forward biased, and the diode-loaded stepped-impedance resonators act as quarter-wavelength resonators. By interlacing the loaded quarter-wavelength resonators with the unloaded half-wavelength resonators to stagger their resonant frequencies for rejecting any passband, an excellent wideband isolation can be obtained while in the off state.

\section{RESONANT CONDITIONS FOR STEPPED-IMPEDANCE RESONATORS WITH DIFFERENT LOADS}

Fig. 1 shows the circuit structure of stepped-impedance resonator with a load $Z_{L}$ at one end. The resonator is composed of two transmission-line sections of different line widths with 


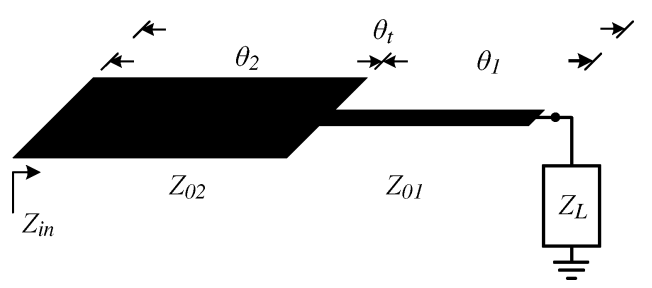

Fig. 1. Layout of the stepped-impedance resonators with loads at one end.

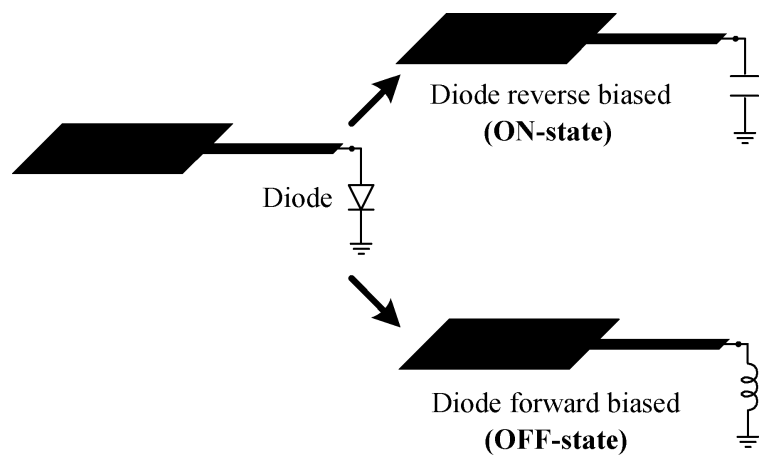

Fig. 2. Stepped-impedance resonator with a diode loaded at one end and its simplified on- and off-state equivalent circuits.

a load $Z_{L}$ connected to one end. If the input impedance of the stepped-impedance resonator seen from the open end is defined as $Z_{\text {in }}$, the parallel resonance of the resonator occurs when $1 / Z_{\text {in }}=Y_{\text {in }}=0$, and the resonance condition may be written as

$Z_{01}\left(Z_{02}-Z_{01} \tan \theta_{1} \tan \theta_{2}\right)+j Z_{L}\left(Z_{02} \tan \theta_{1}+Z_{01} \tan \theta_{2}\right)=0$

where $Z_{01}$ and $Z_{02}$ are the characteristic impedances of the two line sections, and $\theta_{1}$ and $\theta_{2}$ are the electrical lengths of the two line sections.

By defining the length ratio $\alpha$ of the stepped-impedance resonator as

$$
\alpha=\frac{\theta_{1}}{\theta_{1}+\theta_{2}}=\frac{\theta_{1}}{\theta_{t}}
$$

one may rewrite the resonance condition as

$$
\begin{array}{r}
Z_{02}\left[Z_{01}+j Z_{L} \tan \left(\alpha \theta_{t}\right)\right]+j Z_{01}\left[Z_{L}+j Z_{01} \cdot \tan \left(\alpha \theta_{t}\right)\right] \\
\cdot \tan (1-\alpha) \theta_{t}=0 .
\end{array}
$$

In this paper, the switchable resonator is realized by replacing $Z_{L}$ with a p-i-n diode. Fig. 2 shows the proposed circuit configuration of the diode-loaded stepped-impedance resonator and its simplified on- and off-state equivalent circuits. When the diode is reverse-biased (on state), the resonator is loaded with a junction capacitor. If the diode is forward-biased (off state), the resonator is then terminated by a parasitic inductor. Therefore, resonant frequencies of the resonator could be changed by applying different bias conditions to the diode. In the following subsections, the resonance conditions of the stepped-impedance resonators with different loads $Z_{L}$ (inductive or capacitive) will be analyzed and discussed to characterize the resonance phenomenons.

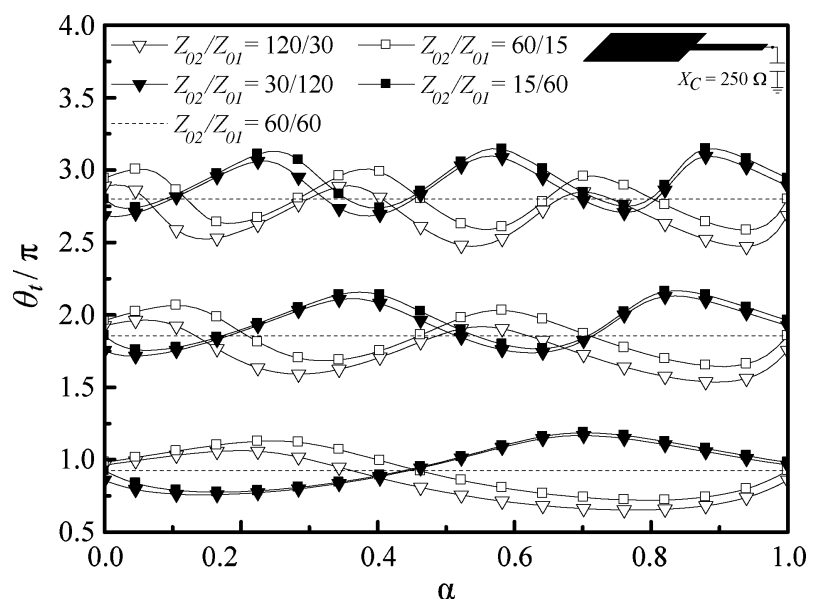

Fig. 3. Resonant conditions for stepped-impedance resonators with capacitive load $X_{C}=250 \Omega$.

\section{A. Capacitive Load}

In beginning, consider the case of capacitive loads. Applying $Z_{L}=-j X_{C}$ to (3) yields

$$
\begin{array}{r}
Z_{02}\left[Z_{01}+X_{C} \tan \left(\alpha \theta_{t}\right)\right]+Z_{01}\left[X_{C}-Z_{01} \cdot \tan \left(\alpha \theta_{t}\right)\right] \\
\cdot \tan (1-\alpha) \theta_{t}=0 .
\end{array}
$$

From (4), it is observed that the resonance condition depends on the stepped length ratio, the impedance values of $Z_{02}$ and $Z_{01}$, and the loaded capacitive reactance. To investigate the resonant condition, Fig. 3 is plotted according to a given capacitive load $\left(X_{C}=250 \Omega\right)$. Note that the capacitive reactance value $X_{C}$ is given at the first resonant frequency $f_{0}$. In Fig. 3, the resonant electrical length $\theta_{t}$ with respect to different line impedance conditions for a given capacitive load are shown. The trends of these curves are similar to those of the resonators with $Z_{L}$ open-circuited, but the required electrical length $\theta_{t}$ for each resonance mode is reduced. This is because the capacitive load absorbs some electrical length of the open end transmission line. In the uniform-impedance cases, the ratio of the $n$th resonant frequency to fundamental frequency $\left(f_{n} / f_{0}\right)$ is slightly greater than $(n+1)$, and the ratio will increase as the line impedance is higher or the capacitive reactance is lower. In the stepped case, under a given capacitive load, one can keep a low-impedance section longer than a high-impedance section under $\alpha>0.5$, then the ratio of $f_{n} / f_{0}$ will be lower than that of its uniform impedance case. On the contrary, if the high-impedance section is longer than the low-impedance section, the ratio will be greater than that of its uniform-impedance case. It is also noted that, when the capacitance equals zero or $X_{C}=\infty$, the case will become stepped-impedance resonators with both ends opened [10].

\section{B. Inductive Load}

For inductive loads, i.e., $Z_{L}=j X_{L}$, (3) is reduced to

$$
\begin{array}{r}
Z_{02}\left[Z_{01}-X_{L} \tan \left(\alpha \theta_{t}\right)\right]-Z_{01}\left[X_{L}+Z_{01} \cdot \tan \left(\alpha \theta_{t}\right)\right] \\
\cdot \tan (1-\alpha) \theta_{t}=0 .
\end{array}
$$




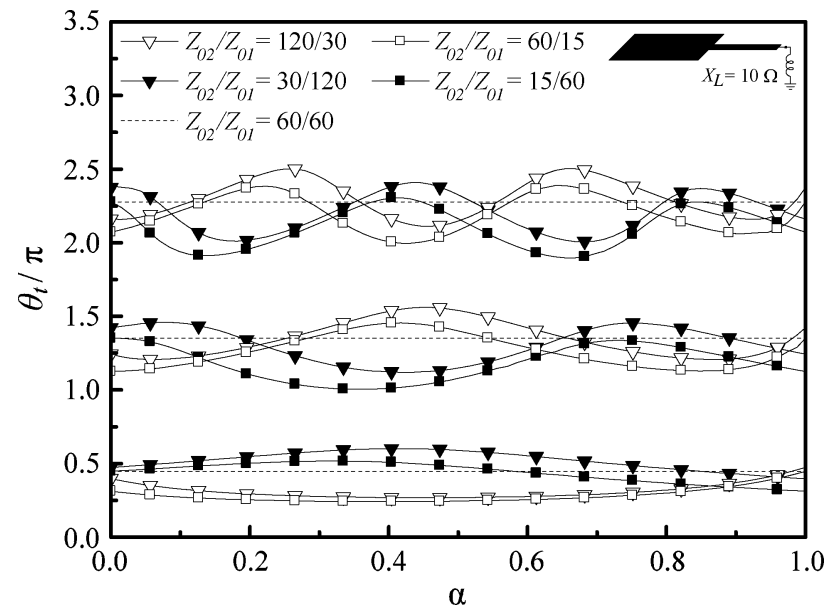

Fig. 4. Resonant conditions for stepped-impedance resonators with inductive loads $X_{L}=10 \Omega$.

The resonance conditions are discussed in Fig. 4 under the inductive load of $X_{L}=10 \Omega$. The inductive reactance value $X_{L}$ is given at the first resonant frequency. The trends of the curves in these figures are similar to those of the resonators with one end short-circuited, but the resonant electrical length needed for each resonance is decreased. Physically, the inductive load absorbs some electrical length of the short-circuit transmission line. For a given inductive load, in the case when $Z_{01}=Z_{02}$, the ratio of the $n$th resonant frequency to fundamental resonant frequency is slightly larger than $(2 n+1)$. Also, the lower the line impedance or the larger the inductive reactance, the larger the ratio will be. When $Z_{01} \neq Z_{02}$, for a fixed inductive load, the ratio of $f_{n} / f_{0}$ will be lower than that of its uniform-impedance case if the high-impedance line section is longer than the lowimpedance section under $\alpha<0.5$. On the contrary, the ratio will be greater than that of its uniform-impedance case as the lowimpedance section is longer than the high-impedance section. It is also noted that, when the inductance equals zero or $X_{L}=0$, the case will become stepped-impedance resonators with one end shorted to ground [13].

According to the above discussions, the resonators with capacitive loads at one end behave like half-wavelength resonators, while those with inductive loads at one end behave like quarter-wavelength resonators. From (4) and (5), the resonance conditions are related to a few parameters. Therefore, there will be flexibility to arrange the resonant frequencies. For example, when a specific capacitive/inductive load is given, one can set the fundamental resonance to a specific frequency and keep the spurious frequencies away from other resonant frequencies of other resonators by properly adjusting the length ratio and impedances of the two line sections.

\section{Electronically Switchable FILTERS Using DIODE-LOADED STEPPED-IMPEDANCE RESONATORS}

Based on the resonant frequencies of loaded resonators, a new concept to design electronically switchable filters using diodeloaded stepped-impedance resonators is proposed. In order to estimate the resonant frequencies of the diode-loaded resonator, the equivalent-circuit model of the p-i-n diode is given in Fig. 5. Here, the Infineon's BAR65-02V p-i-n diode [19] is used, with

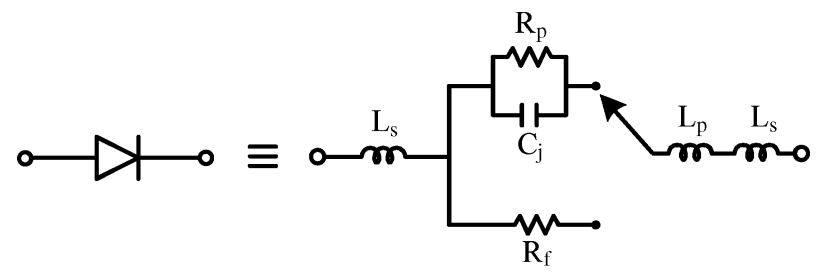

Fig. 5. Equivalent-circuit model of the p-i-n diode.

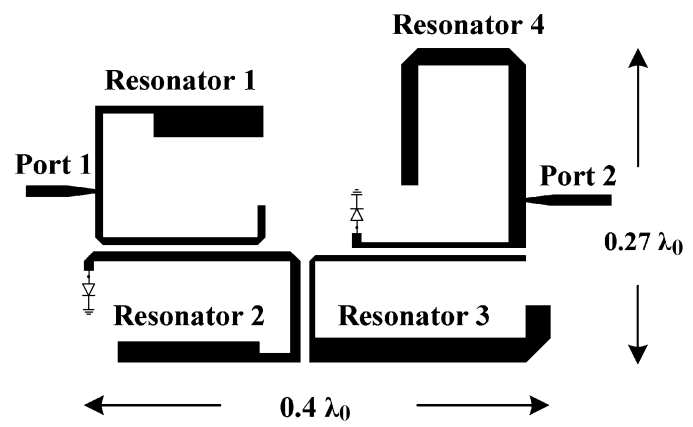

Fig. 6. Layout of the fourth-order switchable filter A composed of two diodeloaded resonators and two unloaded resonators.

$L_{P}=0.8 \mathrm{nH}, L_{S}=0.1 \mathrm{nH}$, junction capacitor $C_{j}=0.33 \mathrm{pF}$ at $-10 \mathrm{~V}$ with reversed parallel resistance $R_{p}=10 \mathrm{k} \Omega$, and forward resistance $R_{f}=1 \Omega$ under 1-mA biasing current. The reverse junction capacitor value varies from 0.34 to $0.33 \mathrm{pF}$ when the bias voltage changes from -6 to $-20 \mathrm{~V}$, and the $Q$-factor of the junction capacitor at $1.5 \mathrm{GHz}$ is approximately 60 . The operating temperature range of the diode is between $-55^{\circ} \mathrm{C}$ and $125^{\circ} \mathrm{C}$.

In the following subsections, two fourth-order Butterworthtype single-pole-single-throw (SPST) switchable bandpass filters using two and three loaded switchable resonators will be demonstrated. For convenience, they are labeled as switchable filters $\mathrm{A}$ and $\mathrm{B}$, respectively.

\section{A. Fourth-Order SPST Switchable Filter With Two Diode-Loaded Stepped-Impedance Resonators}

Fig. 6 shows the circuit configuration of the fourth-order Butterworth microstrip coupled-resonator switchable filter. The diodes connected to the resonators are biased via $10-\mathrm{k} \Omega$ resistors. In the on state (i.e., diodes are reverse-biased), by properly adjusting the impedance and length ratio of the resonator, the four stepped-impedance resonators could be designed to have the same fundamental frequency while with staggered higher order spurious frequencies. As a consequence, the spurious passband of the bandpass filter could be rejected [12], [13]. When the switchable filter is turned off (i.e., diodes are forward-biased), the equivalent terminated loads of resonators 2 and 4 are changed from capacitors to inductors, which means that the resonance conditions of the diode-loaded resonators 2 and 4 are switched from half-wavelength resonators to quarter-wavelength resonators. Thus, under the same geometry structure, the first two resonant frequencies will move from around 1 and 2 times to near 0.5 and 1.5 times the center frequency. Therefore, a high isolation could be obtained around the center frequency. Moreover, the resonant frequencies of the 
TABLE I

Circuit Parameters and Resonant Frequencies of EAch Resonator for Switchable Filter A in the On and OfF States

\begin{tabular}{|c|c|c|c|c|c|c|c|c|c|c|}
\hline & \multirow[b]{2}{*}{$Z_{02} / Z_{01}$} & \multirow[b]{2}{*}{$\theta_{1} /\left(\theta_{1}+\theta_{2}\right)$} & \multicolumn{4}{|c|}{ ON-state } & \multicolumn{4}{|c|}{ OFF-state } \\
\hline & & & $f_{0}$ & $f_{1}$ & $f_{2}$ & $f_{3}$ & $f_{0}$ & $f_{1}$ & $f_{2}$ & $f_{3}$ \\
\hline R1 & $23 / 62$ & 0.78 & 1.50 & 3.45 & 5.43 & 7.16 & 1.50 & 3.45 & 5.43 & 7.16 \\
\hline R2 & $32 / 54$ & 0.72 & 1.50 & 3.28 & 4.94 & 6.36 & 0.71 & 2.44 & 4.25 & 5.75 \\
\hline R3 & $72 / 28$ & 0.48 & 1.50 & 3.04 & 4.48 & 6.04 & 1.50 & 3.04 & 4.48 & 6.04 \\
\hline R4 & $38 / 72$ & 0.27 & 1.50 & 2.61 & 4.09 & 5.67 & 0.59 & 2.12 & 3.75 & 5.02 \\
\hline
\end{tabular}

off-state resonators are also designed to distribute irregularly over the band of interest to achieve a wideband isolation.

The design procedure for a switchable filter could be summarized as follows.

1) Determine the geometric structures of the on-state resonators (diodes are reverse-biased) such that the constitutive stepped-impedance resonators have the same fundamental frequency, but different spurious frequencies.

2) Check the resonant frequencies of off-state steppedimpedance resonators (diodes are forward-biased) if they are misaligned over the band of interest. If not, go back to step 1) and fine tune the geometric structures of the stepped-impedance resonators.

3) Decide the bandpass filter response, using the coupled-resonator filter theory to obtain the distance between coupled resonators and input/output tapping points according to different filter-type responses.

According to the design procedure, the circuit parameters and resonant frequencies of the four stepped-impedance resonators in switchable filter A are listed in Table I. It can be observed that, in the on state, each resonator has the same fundamental resonant frequency of $1.5 \mathrm{GHz}$ and different higher order resonant frequencies in order to reject spurious response. In the off state, the resonant frequencies of the four resonators are also misaligned to achieve good isolation.

The fourth-order Butterworth-type bandpass filter was designed to have a center frequency of $1.5 \mathrm{GHz}$ and $3-\mathrm{dB}$ fractional bandwidth $\Delta_{3 \mathrm{~dB}}$ of $5 \%$. For the given specifications, the coupling coefficients and the external quality factor $Q_{\text {ext }}$ can be obtained as

$$
\begin{aligned}
M_{12} & =\frac{\Delta_{3 \mathrm{~dB}}}{\sqrt{g_{1} g_{2}}}=M_{34}=0.042 \\
M_{23} & =\frac{\Delta_{3 \mathrm{~dB}}}{\sqrt{g_{2} g_{3}}}=0.027 \\
Q_{\mathrm{ei}} & =\frac{g_{0} g_{1}}{\Delta_{3 \mathrm{~dB}}}=Q_{\text {eo }}=15.3
\end{aligned}
$$

where $M_{i j}$ represents the coupling coefficient between resonators $i$ and $j, g_{n}$ is the low-pass prototype parameter, and $Q_{\mathrm{ei}}$ and $Q_{\mathrm{eo}}$ are the external quality factors in association with the input and output couplings, respectively [20].

The proposed switchable filter is fabricated on a Rogers RO4003 substrate with a dielectric constant of 3.38, thickness of $0.508 \mathrm{~mm}$, and loss tangent of 0.0028 . Fig. 6 shows the layout of the fourth-order switchable filter $\mathrm{A}$. The dimension of this circuit is $0.4 \lambda_{0}$ by $0.27 \lambda_{0}\left(49 \times 33 \mathrm{~mm}^{2}\right)$, where $\lambda_{0}$ is the guided wavelength of the microstrip line on this substrate at

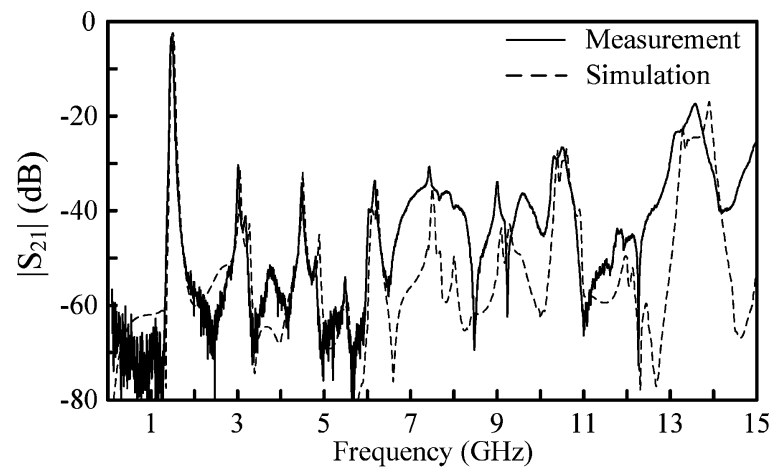

(a)

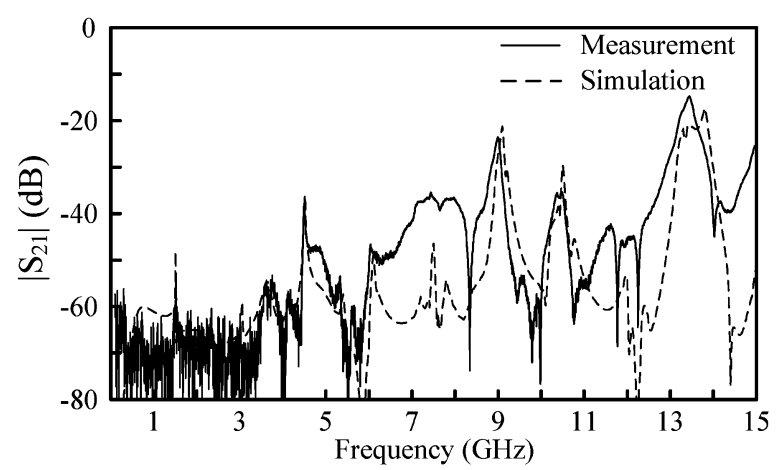

(b)

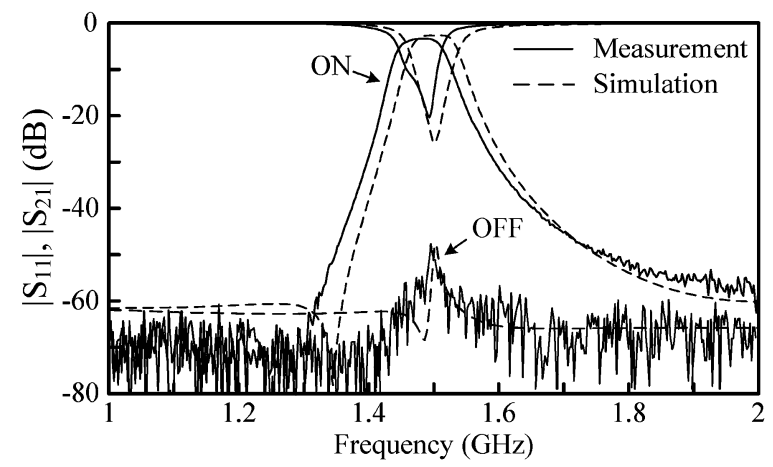

(c)

Fig. 7. Measured and simulated performance of the switchable filter A. (a) On state. (b) Off state. (c) Narrowband response.

the center frequency. The electromagnetic simulation tool used here is Sonnet [21].

Fig. 7 shows the measured and simulated results of the switchable filter A. At the on state, the circuit performs a bandpass filter function with a measured passband insertion loss of $3.4 \mathrm{~dB}$ at a center frequency of $1.49 \mathrm{GHz}$ with a $3-\mathrm{dB}$ 


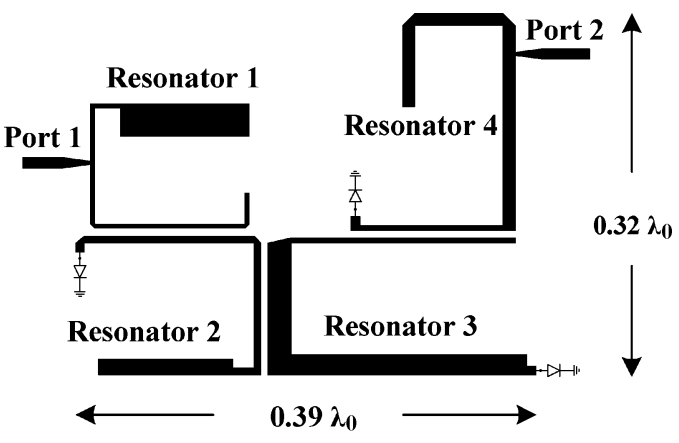

Fig. 8. Layout of the fourth-order switchable filter B composed of three diodeloaded resonators and one unloaded resonator.

bandwidth of $5 \%$. It is noted that some additional passband insertion loss is resulted from the finite $Q$-factor of the junction capacitor when the diodes are reversed-biased. It is also observed that, in the on state, the filter has a wide stopband rejection of $30 \mathrm{~dB}$ up to $10.5 \mathrm{GHz}$. In the off state, the circuit has a measured isolation of $47 \mathrm{~dB}$ at the center frequency and a wideband isolation better than $35 \mathrm{~dB}$ from dc to $8.7 \mathrm{GHz}$.

In designing a conventional switch, the performances of a switch highly depend on the device characteristics. In other words, a high-performance switch needs both a small junction capacitor $\left(C_{j}\right)$ and a small on-state resistor $\left(R_{f}\right)$; however, there is always a tradeoff in obtaining a small $C_{j}$ and a small $R_{f}$, either for diodes or passive FETs. Unlike the conventional switches, the $C_{j}$ of the proposed switchable filter serves as a capacitive load of the stepped impedance resonator in the thru-state, and the value of $C_{j}$ will not affect the passband insertion loss. In the off state, the proposed switchable filters achieve high isolations by staggering the resonant frequencies of each resonator, which means that the high isolation is not mainly determined by the value of the loaded forward resistor $R_{f}$.

One thing worth mentioning is that conventional switches using p-i-n diodes usually need high forward biasing current for small turn-on resistance to minimize insertion losses or achieve high isolations, but that will result in a high dc power dissipation. The diodes of the proposed switchable filters only need to be biased in barely turn-on region, under which the total dc power consumption is less than $1 \mathrm{~mW}$.

\section{B. Fourth-Order SPST Switchable Filter With Three Diode-Loaded Stepped-Impedance Resonators}

In the previous design, when switchable filter $\mathrm{A}$ is switched off, the two unloaded resonators, resonators 1 and 3 , have the same fundamental frequency $f_{0}$ of $1.5 \mathrm{GHz}$. Therefore, a little spike at the center frequency in the off state is observed in Fig. 7(c) from both the simulation and measurement results. Intuitively, if we can avoid the two resonators resonating at the center frequency in the off state, a higher isolation could be obtained around the center frequency. This idea could be easily realized by using three diode-loaded resonators together with one unloaded resonator in a fourth-order design.

Fig. 8 shows the circuit configuration of the microstrip coupled-resonator switchable filter B. In this design example, the fourth-order switchable filter B is constructed by three diode-loaded stepped-impedance resonators together with one unloaded stepped-impedance resonator. The circuit parameters and resonant frequencies of the four resonators are listed in Table II. In the on state, the four stepped-impedance resonators have the same $f_{0}$ of $1.5 \mathrm{GHz}$ and different spurious frequencies. In the off state, the resonant frequencies of the four stepped-impedance resonators are scattered over the band of interest to achieve good isolation.

Switchable filter B was designed to have a Butterworth-type response at a center frequency of $1.5 \mathrm{GHz}$ and $5 \%$ bandwidth. The required coupling coefficients and external quality factors can be found in (6) for the same specification. The circuit was also fabricated on the same Rogers RO4003 substrate. The physical dimensions of the circuit is $0.39 \lambda_{0} \times 0.32 \lambda_{0}\left(50 \times 40 \mathrm{~mm}^{2}\right)$.

Fig. 9 shows the measured and simulated results. In the on state, the switchable filter B presents a bandpass filter response with a measured passband insertion loss of $3.9 \mathrm{~dB}$ at a center frequency of $1.49 \mathrm{GHz}$ with $3-\mathrm{dB}$ fractional bandwidth of $5 \%$, and it has a stopband rejection of $30 \mathrm{~dB}$ up to $13.5 \mathrm{GHz}$. As can be predicted, the passband insertion loss is higher than that of the switchable filter A due to the use of three diode-loaded resonators instead of two. When switched off, the circuit shows a measured isolation of about $65 \mathrm{~dB}$ at the center frequency, which has an improvement of $18 \mathrm{~dB}$ compared with that of the switchable filter A. It also presents a wideband isolation of better than $35 \mathrm{~dB}$ from dc to $13.3 \mathrm{GHz}$. According to the results, the tradeoffs between the passband insertion loss and in-band isolation depend on the different number of diode-loaded steppedimpedance resonators used in each design.

From the above discussions, most design efforts were focused on determining geometric structures of the resonators. Sometimes a few iterations may be needed, then (4), (5), and their corresponding figures can serve as guidelines to fine tune the circuit.

\section{COMPACT SPDT SWITCHABLE FILTER WITH COMMON RESONATORS}

In modern TDD wireless communication systems, an SPDT switch and a preselected bandpass filters are essential building blocks at the RF front-end, as shown in Fig. 10(a). Based on the SPST switchable filter described above, a concept of integrating a preselected bandpass filter with an SPDT switch into a single circuit is illustrated in Fig. 10(b).

The SPDT switchable filter is designed on the basis of two SPST ones. Fig. 11(a) shows the conventional coupling structure of the SPDT circuit, where each solid dot represents an unloaded resonator, each hollow circle represents a diode-loaded resonator, and the solid lines indicate the main path couplings. The input-matching $\mathrm{T}$-junction is used to transform the input impedance of isolated terminal into high impedance at the center frequency, which is widely used in the millimeter-wave SPDT switch and diplexer circuit designs [4], [5], [17], [18]. Fig. 11(b) shows the proposed coupling structure for designing a compact SPDT switchable filter: the structure utilizes two common resonators to replace the matching $\mathrm{T}$-junction and reduce the total number of resonators. As a result, the total circuit size could be significantly reduced.

The circuit structure of this SPDT switchable filter is shown in Fig. 12. In later discussions, the resonators in the circuit are 
TABLE II

Circuit Parameters and Resonant Frequencies of Each Resonator for Switchable Filter B in the On and OfF States

\begin{tabular}{|c|c|c|c|c|c|c|c|c|c|c|}
\hline & \multirow[b]{2}{*}{$Z_{02} / Z_{01}$} & \multirow[b]{2}{*}{$\theta_{1} /\left(\theta_{1}+\theta_{2}\right)$} & \multicolumn{4}{|c|}{ ON-state } & \multicolumn{4}{|c|}{ OFF-state } \\
\hline & & & $f_{0}$ & $f_{1}$ & $f_{2}$ & $f_{3}$ & $f_{0}$ & $f_{1}$ & $f_{2}$ & $f_{3}$ \\
\hline $\mathrm{R} 1$ & $22 / 78$ & 0.72 & 1.50 & 3.72 & 5.57 & 6.81 & 1.50 & 3.72 & 5.57 & 6.81 \\
\hline $\mathrm{R} 2$ & $37 / 62$ & 0.72 & 1.50 & 4.95 & 6.39 & 8.00 & 0.72 & 2.48 & 4.32 & 5.82 \\
\hline R3 & $72 / 31$ & 0.61 & 1.50 & 2.43 & 4.09 & 5.31 & 0.80 & 1.89 & 3.05 & 4.63 \\
\hline R4 & $44 / 72$ & 0.28 & 1.50 & 2.71 & 5.77 & 7.01 & 0.63 & 2.19 & 3.80 & 5.14 \\
\hline
\end{tabular}

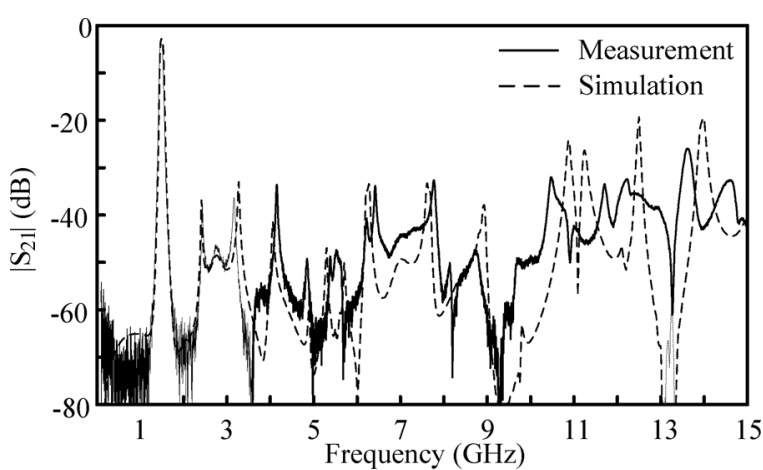

(a)

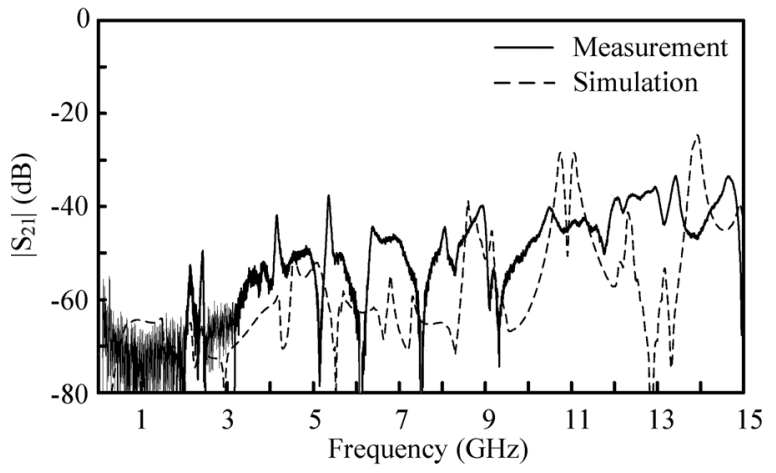

(b)

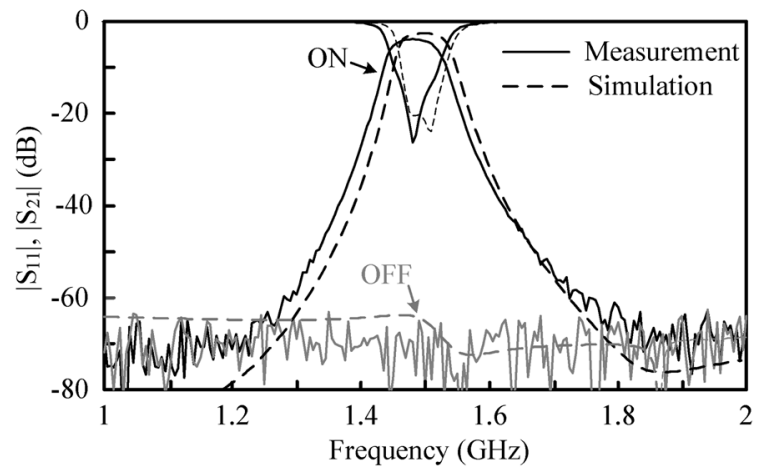

(c)

Fig. 9. Measured and simulated performance of the switchable filter B. (a) On state. (b) Off state. (c) Narrowband response.

labeled R1-R6 for convenience. As can be observed, the two unloaded R1 and R2 are used as the common resonators, and the diode-loaded resonators R3-R6 are used to control the signal flow. Consider the condition when R3 and R4 are on (the loaded diodes are reverse-biased) and R5 and R6 are off (the loaded
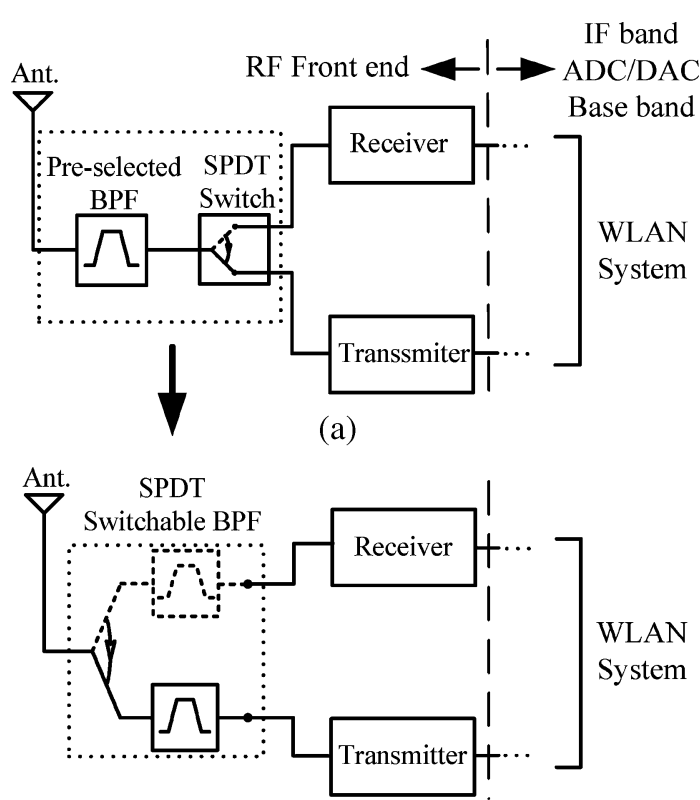

(b)

Fig. 10. System block of a TDD wireless communication system. (a) Conventional architecture using a preselected bandpass filter and a SPDT switch. (b) Proposed architecture using an SPDT electronically switchable bandpass filter.

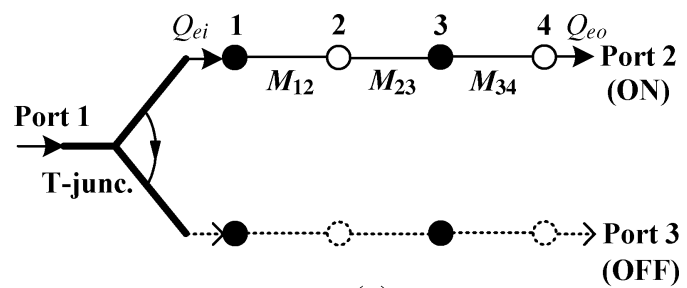

(a)

(OFF)

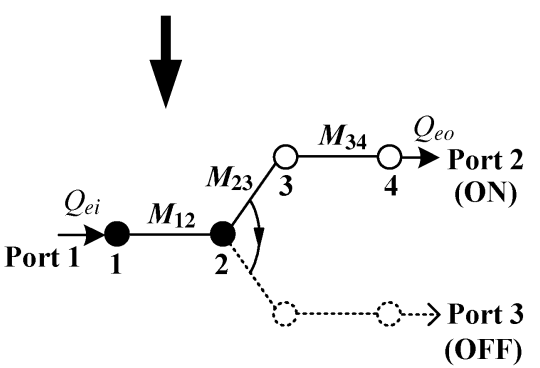

(b)

Fig. 11. Coupling structures of a fourth-order SPDT switchable filter. (a) Conventional structure. (b) Proposed structure. 


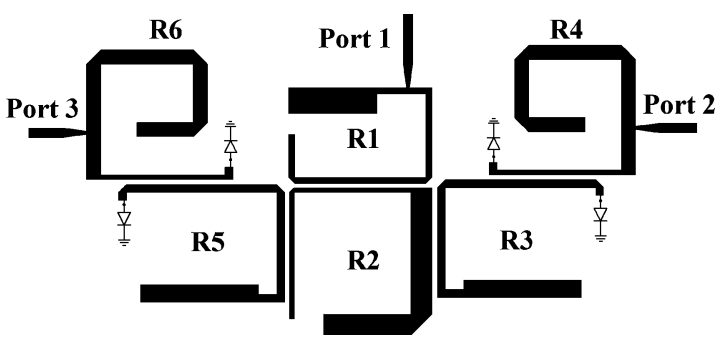

Fig. 12. Layout of the SPDT switchable filter using mutually coupled resonators.

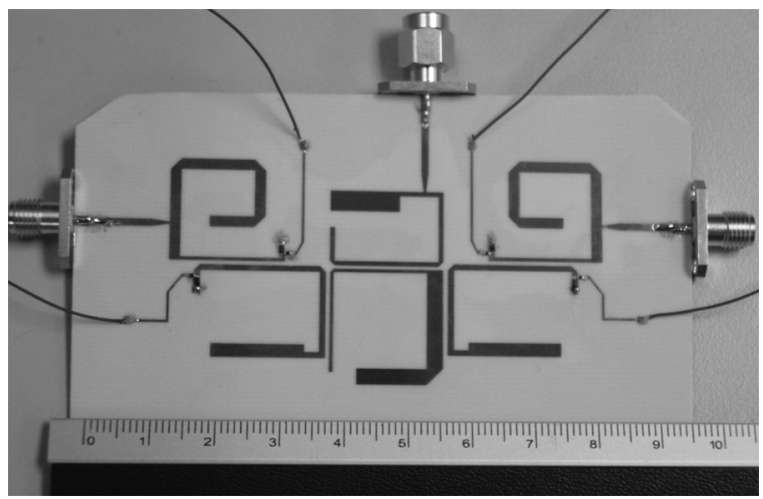

Fig. 13. SPDT switchable filter

diodes are forward-biased). As a consequence, R1-R4 will form a fourth-order bandpass filter to allow the passband signal to pass from port 1 to port 2 . In the meanwhile, the passband of interest is not affected by the presence of resonators R5 and R6 because the resonant frequencies of the off-state resonators do not locate in the vicinity of the center frequency. Note that, due to misalignments of resonance frequencies of R1-R2 and R5-R6, port 3 stays in an isolated state. If the bias condition is reversed, then the passband signal will pass through port 3 , and port 2 will be in the isolated state. In this SPDT circuit design, the two SPST circuits can be designed independently. This means that the filter composed of R1-R4 and the filter composed of R1-R2 and R5-R6 could follow the SPST design flow as mentioned before and then combine the two SPST circuits by means of the unloaded common resonators to accomplish an SPDT switchable filter.

The SPDT switchable filter was also fabricated on the same RO4003 substrate. Fig. 13 shows the photograph of the SPDT switchable filter. Fig. 14 shows the measured performance of this circuit. During testing, only one port was set at the thrustate, while the other output port stayed at the isolated state. In Fig. 14(a), at the thru-port (port 2), a bandpass response with a passband insertion loss of $3.6 \mathrm{~dB}$ at a center frequency of $1.49 \mathrm{GHz}$ with $3-\mathrm{dB}$ fractional bandwidth of $5 \%$ was measured, and a stopband rejection of $30 \mathrm{~dB}$ up to $15 \mathrm{GHz}$ was obtained. At the isolated-port (port 3), the circuit shows a measured isolation greater than $47 \mathrm{~dB}$ at the center frequency, and a wideband isolation is better than $35 \mathrm{~dB}$ from dc to $10.4 \mathrm{GHz}$. Fig. 14(b) shows the measured results when port 3 is on and port 2 is off. The measured passband insertion loss of port 3 is $3.6 \mathrm{~dB}$ at $1.49 \mathrm{GHz}$ with a 3-dB fractional bandwidth of $5 \%$, and a stopband rejection of $27 \mathrm{~dB}$ up to $15 \mathrm{GHz}$ was also obtained. The measured isolation at port 2 is better than $48 \mathrm{~dB}$ at the center

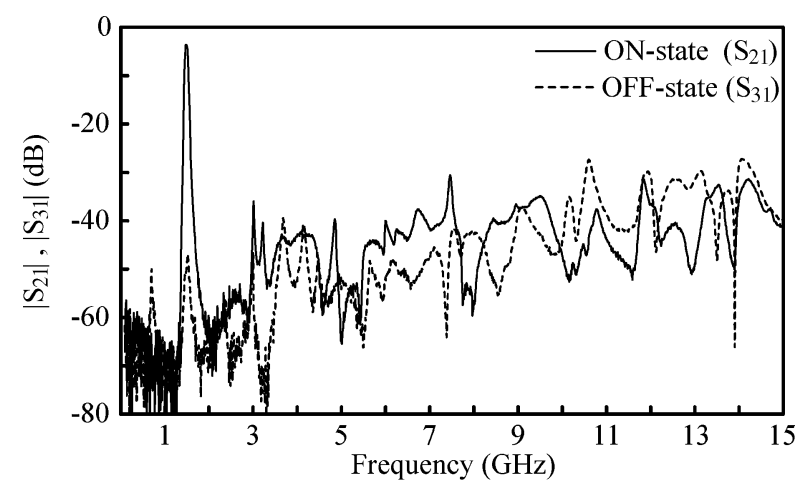

(a)

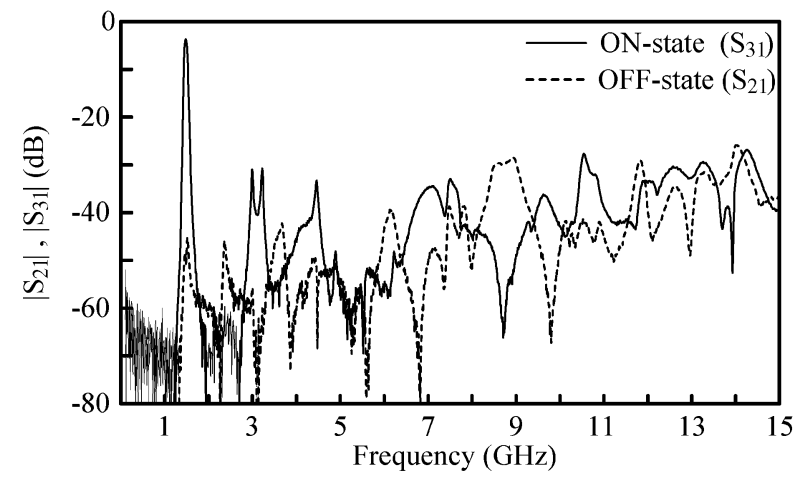

(b)

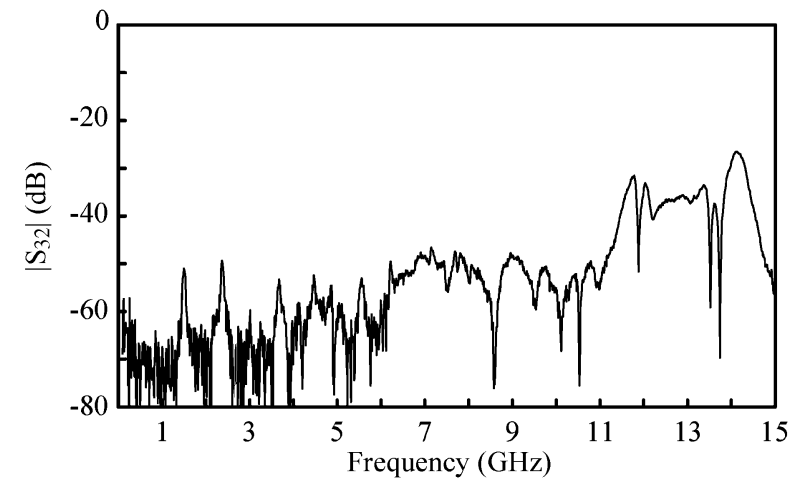

(c)

Fig. 14. Measured results of the SPDT switchable filter. (a) On-/off-state $\left(S_{21} / S_{31}\right)$. (b) Leakage between transmitter and receiver $\left(S_{32}\right)$. (c) Narrowband response.

frequency, and a wideband isolation of better than $35 \mathrm{~dB}$ from dc to $8.4 \mathrm{GHz}$ was also observed. Fig. 14(c) shows the isolation between ports 2 and 3, which represents the leakage from the transmitter to the receiver ends. The measured results shows that the leakage is $51 \mathrm{~dB}$ at the center frequency and higher than $45 \mathrm{~dB}$ from dc to $11.3 \mathrm{GHz}$.

In this fourth-order SPDT switchable filter design, two common resonators are utilized to reduce the number of total resonators. Actually, the number of common resonators equals the unloaded resonators used in each SPST switchable filter design. For example, if three common resonators are used in this SPDT design, the total number of resonators will be reduced to five, but the isolation performance will degrade due to the fact that there is only one switchable resonator in each signal path. On the contrary, if only one common resonator is used (three switchable resonators will exist in each signal path), 
TABLE III

COMPARISON TABLE

\begin{tabular}{|c|c|c|c|c|c|c|}
\hline \multirow[b]{2}{*}{ Ref. } & \multirow[b]{2}{*}{ Technology } & \multirow[b]{2}{*}{ Topology } & \multicolumn{3}{|c|}{ ON-state } & \multirow{2}{*}{$\begin{array}{c}\text { OFF-state } \\
\text { Passband isolation } \\
\text { (dB) }\end{array}$} \\
\hline & & & $\begin{array}{c}\text { 3-dB BW } \\
(\%)\end{array}$ & $\begin{array}{c}\mathrm{IL}^{\dagger} \text { at } f_{0} \\
(\mathrm{~dB} \text { at } \mathrm{GHz})\end{array}$ & $\begin{array}{c}\text { Spurious suppress } \\
(\mathrm{dB})\end{array}$ & \\
\hline Switch [1] & $\begin{array}{l}0.5 \mu \mathrm{m} \\
\mathrm{CMOS}\end{array}$ & series shunt & N.A. & 0.7 at 0.9 & - & $>40$ from 0.8 to $1 \mathrm{GHz}$ \\
\hline Switch [2] & $\begin{array}{l}0.25 \mu \mathrm{m} \mathrm{SOI} \\
\mathrm{CMOS}\end{array}$ & series shunt & N.A. & $\begin{array}{l}0.7 \text { at } 2.4 \\
1.0 \text { at } 5.0\end{array}$ & - & $>46$ from dc to $5 \mathrm{GHz}$ \\
\hline Switch [3] & $\begin{array}{c}0.13 \mu \mathrm{m} \\
\mathrm{CMOS}\end{array}$ & series shunt & N.A. & 1.8 at 15 & - & $>17.8$ from 7 to $15 \mathrm{GHz}$ \\
\hline Filter [11] & Duroid 4003 & $\begin{array}{c}\text { 3rd-order, } \\
\text { coupled-line }\end{array}$ & 10 & 1.5 at 0.9 & 47 up to $3 f_{0}$ & - \\
\hline Filter [12] & Duroid 4003 & $\begin{array}{c}\text { 4th-order, } \\
\text { coupled-resonator }\end{array}$ & 4.5 & 2.7 at 1.5 & 30 up to $8.4 f_{0}$ & - \\
\hline Filter [13] & Duroid 4003 & $\begin{array}{c}\text { 4th-order, } \\
\text { coupled-resonator }\end{array}$ & 7.6 & 2.6 at 2.0 & 27.5 up to $11.4 f_{0}$ & - \\
\hline Switchable filter [14] & $\begin{array}{c}\text { pin diode, } \\
\text { Duroid } 5880\end{array}$ & $\begin{array}{l}\text { 2nd-order, } \\
\text { ring filter }\end{array}$ & 11 & 2.0 at 2.6 & - & 25 \\
\hline Switchable filter [15] & $\begin{array}{c}\text { pin diode, } \\
\text { Duroid } 6010\end{array}$ & $\begin{array}{l}\text { 3rd-order, } \\
\text { stub filter }\end{array}$ & 13 & 1.7 at 3.0 & - & 25 \\
\hline Switchable filter [16] & $\begin{array}{c}\text { HJFET, } \\
\text { FR4 }\end{array}$ & $\begin{array}{l}\text { 5th-order, } \\
\text { stub filter }\end{array}$ & 60 & 1.3 at 1.0 & - & 22 \\
\hline $\begin{array}{c}\text { Switchable filter A } \\
\text { (this work) }\end{array}$ & $\begin{array}{c}\text { pin diode, } \\
\text { Duroid } 4003\end{array}$ & $\begin{array}{c}\text { 4th-order, } \\
\text { coupled-resonator }\end{array}$ & 5 & 3.4 at 1.49 & 30 up to $7 f_{0}$ & $47,>35$ from dc to $5.8 f_{0}$ \\
\hline $\begin{array}{c}\begin{array}{c}\text { Switchable filter B } \\
\text { (this work) }\end{array} \\
\end{array}$ & $\begin{array}{c}\text { pin diode, } \\
\text { Duroid } 4003\end{array}$ & $\begin{array}{c}\text { 4th-order, } \\
\text { coupled-resonator }\end{array}$ & 5 & 3.9 at 1.49 & 30 up to $9 f_{0}$ & $65,>35$ from dc to $8.9 f_{0}$ \\
\hline $\begin{array}{l}\text { SPDT Switchable filter } \\
\text { (this work) }\end{array}$ & $\begin{array}{c}\text { pin diode, } \\
\text { Duroid } 4003\end{array}$ & $\begin{array}{c}\text { 4th-order, } \\
\text { coupled-resonator }\end{array}$ & 5 & 3.6 at 1.49 & 30 up to $10 f_{0}$ & $47,>35$ from dc to $7.0 f_{0}$ \\
\hline
\end{tabular}

${ }^{\ddagger} \mathrm{BW}=$ Bandwidth.

${ }^{\dagger} \mathrm{IL}=$ Insertion loss

then the isolation performance can be improved with a tradeoff for the circuit size and passband insertion loss. Therefore, for a compromise among the circuit size, passband insertion loss, and isolation, two common resonators are adopted in this SPDT design.

To compare direct cascade of a conventional filter and a switch with the proposed switchable filters, Table III shows recently published wide stopband filters, high-performance monolithic-microwave integrated-circuit (MMIC) switches, and electronically switchable filters. At the on state, the proposed switchable filters serve as bandpass filters with good stopband rejection which are comparable to the filters with wide stopband performances [11]-[13]. At the off state, the proposed switchable filters provide high and wideband isolations that are competitive with those of MMIC switches [1]-[3]. In other words, the proposed switchable filters can have both the advantages of a high-performance switch and a bandpass filter with good stopband rejection. It is observed that the previously published switchable filters could not provide a good stopband rejection as a bandpass filter at the on state and wideband isolation at the off state [14]-[16]. Compare with the bandpass filters with the same order and 3-dB bandwidth [12]: the passband insertion losses increased is about $1 \mathrm{~dB}$, while it can trade for high and wideband isolations in the off state. By embedding the diodes into the stepped-impedance resonators to implement the switchable filters, another advantage is to eliminate a switch. The advantage will be more obvious at higher frequencies when the size of a switch is comparable to that of a filter [4], [5]. These features will be useful for many system applications and high-level integration MMICs.

\section{CONCLUSION}

A new concept to design electronically switchable filters using diode-loaded stepped-impedance resonators is proposed in this paper. Resonance conditions of stepped-impedance resonators with different loads at one end are also studied and discussed. The proposed switchable filters successfully integrate a bandpass filter and a switch into a single component and can combine both of their advantages. Besides the wide stopband rejection of the bandpass filter response in the on state, high isolation performance is also obtained from dc to many octave bandwidth in the off state. Finally, a compact SPDT switchable filter using common resonators is also demonstrated to show its application in wireless communication systems. Although the design concept is demonstrated using hybrid circuits in this paper, the idea could also be easily applied to MMIC design for high-level integrations.

\section{ACKNOWLEDGMENT}

The authors would like to thank M.-I. Lai and R.-B. Sun, both with National Taiwan University, Taipei, Taiwan, R.O.C., for the helpful suggestions.

\section{REFERENCES}

[1] F. J. Huang and K. O., "A 0.5- $\mu \mathrm{m}$ CMOS T/R switch for 900-MHz wireless applications," IEEE J. Solid-State Circuits, vol. 36, no. 3, pp. 486-492, Mar. 2001 
[2] C. Tinella, J. M. Fournier, D. Belot, and V. Knopik, "A high-performance CMOS-SOI antenna switch for the 2.5-5-GHz band," IEEE $J$. Solid-State Circuits, vol. 38, no. 7, pp. 1279-1283, Jul. 2003.

[3] Z. Li and K. K. O., "15-GHz fully integrated nMOS switches in a 0.13$\mu \mathrm{m}$ CMOS process," IEEE J. Solid-State Circuits, vol. 40, no. 11, pp. 2323-2328, Nov. 2005.

[4] J. Kim, W. Ko, S. H. Kim, J. Jeong, and Y. Kwon, "A high-performance 40-85 GHz MMIC SPDT switch using FET-integrated transmission line structure," IEEE Microw. Wireless Compon. Lett., vol. 13, no. 12, pp. 505-507, Dec. 2003.

[5] K. Y. Lin, W. H. Tu, P. Y. Chen, H. Chen, H. Wang, and R. B. $\mathrm{Wu}$, "Millimeter-wave MMIC passive HEMT switches using traveling-wave concept," IEEE Trans. Microw. Theory Tech., vol. 52, no. 8, pp. 1798-1808, Aug. 2004.

[6] S. B. Cohn, "Parallel coupled transmission-line resonator filters," IRE Trans. Microw. Theory Tech., vol. MTT-6, no. 2, pp. 223-231, Apr. 1958.

[7] E. G. Cristal and S. Frankel, "Hairpin-line and hybrid hairpin-line/halfwave parallel-coipled-line filters," IEEE Trans. Microw. Theory Tech., vol. MTT-20, no. 11, pp. 719-728, Nov. 1972.

[8] J. G. Garca, F. Martn, F. Falcone, J. Bonache, I. Gil, T. Lopetegi, M. A. G. Laso, M. Sorolla, and R. Marqus, "Spurious passband suppression in microstrip coupled line bandpass filters by means of split ring resonators," IEEE Microw. Wireless Compon. Lett., vol. 14, no. 9, pp. 416-418, Sep. 2004.

[9] T. Lopetegi, M. A. G. Laso, F. Falcone, F. Martin, J. Bonache, J. Garcia, L. Perez-Cuevas, M. Sorolla, and M. Guglielmi, "Microstrip wigglyline bandpass filters with multispurious rejection," IEEE Microw. Wireless Compon. Lett., vol. 14, no. 11, pp. 531-533, Nov. 2004.

[10] K. F. Chang and K. W. Tam, "Miniaturized cross-coupled filter with second and third spurious responses suppression," IEEE Microw. Wireless Compon. Lett., vol. 15, no. 2, pp. 122-124, Feb. 2005.

[11] P. Cheong, S. W. Fok, and K. W. Tam, "Miniaturized parallel coupledline bandpass filter with spurious-response suppression," IEEE Trans. Microw. Theory Tech., vol. 53, no. 5, pp. 1810-1816, May 2005.

[12] C. F. Chen, T. Y. Huang, and R. B. Wu, "Design of microstrip bandpass filters with multiorder spurious-mode suppression," IEEE Trans. Microw. Theory Tech., vol. 53, no. 12, pp. 3788-3793, Dec. 2005.

[13] S. C. Lin, P. H. Deng, Y. S. Lin, C. H. Wang, and C. H. Chen, "Wide-stopband microstrip bandpass filters using dissimilar quarter-wavelength stepped-impedance resonators," IEEE Trans. Microw. Theory Tech., vol. 54, no. 3, pp. 1011-1018, Mar. 2006.

[14] T. S. Martin, F. Wang, and K. Chang, "Theoretical and experimental investigation of novel varactor-tuned switchable microstrip ring resonator circuits," IEEE Trans. Microw. Theory Tech., vol. 36, no. 12, pp. 1733-1739, Dec. 1988.

[15] Y. H. Shu, J. A. Navarro, and K. Chang, "Electronically switchable and tunable coplanar waveguide-slotline bandpass filters," IEEE Trans. Microwa.Theory Tech., vol. 39, no. 3, pp. 548-554, Mar. 1991.

[16] J. Lee, Z. M. Tsai, and H. Wang, "A bandpass filter-integrated switch using field-effect transistors and its power analysis," in IEEE MTT-S Int. Microw. Symp. Dig., Jun. 2006, pp. 768-771.

[17] B. Strassner and K. Chang, "Wide-band low-loss high-isolation microstrip periodic-stub diplexer for multiple-frequency applications," IEEE Trans. Microw. Theory Tech., vol. 49, no. 10, pp. 1818-1820, Oct. 2001.

[18] S. Srisathit, S. Patisang, R. Phromloungsri, S. Bunnjaweht, S. Kosulvit, and $\mathrm{M}$. Chongcheawchamnan, "High isolation and compact size microstrip hairpin diplexer," IEEE Microw. Wireless Comp. Lett., vol. 15, no. 2, pp. 101-103, Febuary 2005.

[19] "Datasheet for BAR65 Series," Infineon, Munich, Germany. [Online]. Available: http://www.infineon.com/

[20] J. S. Hong and M. J. Lancaster, Microstrip Filter for RF/Microwave Applications. New York: Wiley, 2001, ch. 8.

[21] “Sonnet User's Manual, Release 10.0," Sonnet Software Inc., North Syracuse, NY, 2004.

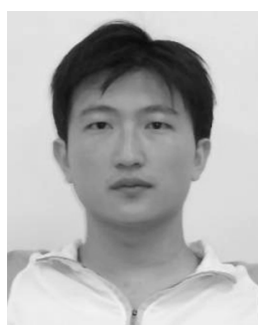

Shih-Fong Chao was born in ChangHua, Taiwan, R.O.C., in 1979. He received the B.S. degree in electrical engineering from National Cheng-Kung University, Tainan, Taiwan, R.O.C., 2002, and is currently working toward the Ph.D. degree at National Taiwan University, Taipei, Taiwan, R.O.C.

His research interests include microwave and millimeter-wave circuit designs.

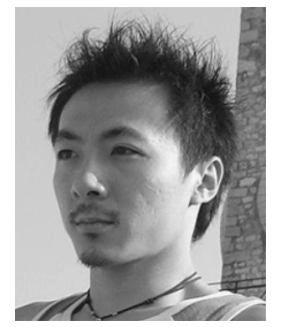

Chao-Huang Wu was born in Taoyuan, Taiwan, R.O.C, in 1980. He received the B.S. degree in electrical engineering from the National Sun Yet-Sen University, Kaohsiung, Taiwan, R.O.C., in 2002, and the Ph.D. degree from the Graduate Institute of Communication Engineering, National Taiwan University, Taipei, Taiwan, R.O.C., in 2006.

His research interests include the design and analysis of microwave filter circuits.

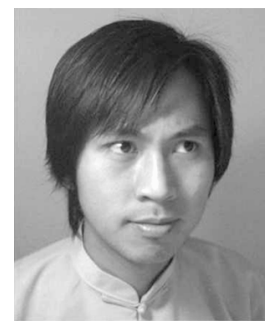

Zou-Min Tsai (S'01) was born in MaioLi, Taiwan, R.O.C., in 1979. He received the B.S. and Ph.D. degrees from the National Taiwan University, Taipei, Taiwan, R.O.C., in 2001 and 2006, respectively.

$\mathrm{He}$ is currently a Post-Doctoral Research Fellow with the Graduate Institute of Communication Engineering, National Taiwan University. His research interest is the theory of microwave circuits.

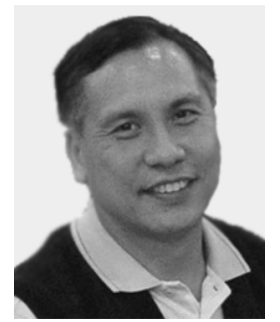

Huei Wang (S'83-M'87-SM'95-F'06) was born in Tainan, Taiwan, R.O.C., on March 9, 1958. He received the B.S. degree from National Taiwan University, Taipei, Taiwan, R.O.C., in 1980, and the M.S. and Ph.D. degrees from Michigan State University, East Lansing, in 1984 and 1987, respectively, all in electrical engineering.

During his graduate studies, he was engaged in research on theoretical and numerical analysis of electromagnetic radiation and scattering problems. $\mathrm{He}$ was also involved in the development of microwave remote detecting/sensing systems. He joined Electronic Systems and Technology Division, TRW Inc., in 1987. He has been an MTS and Staff Engineer responsible for MMIC modeling of CAD tools and MMIC testing evaluation and design, and he became the Senior Section Manager of the MMW Sensor Product Section, RF Product Center, TRW Inc. He visited the Institute of Electronics, National Chiao-Tung University, Hsin-Chu, Taiwan, R.O.C., in 1993 to teach MMIC-related topics and returned to TRW Inc. in 1994. He joined the faculty of the Department of Electrical Engineering, National Taiwan University, as a Professor in February 1998.

Dr. Wang is a member of Phi Kappa Phi and Tau Beta Pi. He was the recipient of the Distinguished Research Award of National Science Council, R.O.C. (2003-2006). He was also elected as the first Richard M. Hong Endowed Chair Professor of National Taiwan University in 2005.

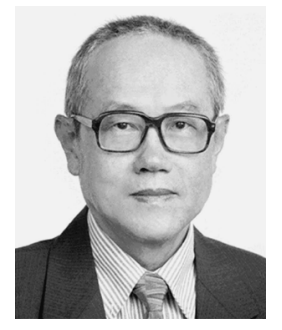

Chun Hsiung Chen (SM'88-F'96) was born in Taipei, Taiwan, R.O.C., on March 7, 1937. He received the B.S.E.E. degree in electrical engineering from National Taiwan University, Taipei, Taiwan, R.O.C., in 1960, the M.S.E.E. degree from National Chiao Tung University, Hsinchu, Taiwan, R.O.C., in 1962, and the Ph.D. degree in electrical engineering from National Taiwan University in 1972.

In 1963, he joined the Faculty of the Department of Electrical Engineering, National Taiwan University, where he is currently a Professor. From August 1982 to July 1985, he was Chairman of the Department of Electrical Engineering, National Taiwan University. From August 1992 to July 1996, he was the Director of the University Computer Center, National Taiwan University. In 1974 he was a Visiting Scholar with the Department of Electrical Engineering and Computer Sciences, University of California at Berkeley. From August 1986 to July 1987, he was a Visiting Professor with the Department of Electrical Engineering, University of Houston, Houston, TX. In 1989, 1990, and 1994, he visited the Microwave Department, Technical University of Munich, Munich, Germany, the Laboratoire d'Optique Electromagnetique, Faculte des Sciences et Techniques de Saint-Jerome, Universite d'Aix-Marseille III, Marseille, France, and the Department of Electrical Engineering, Michigan State University, East Lansing, respectively. His areas of interest include microwave circuits and computational electromagnetics. 